\title{
Impact of epispadias repair on bladder growth in boys with classic bladder exstrophy
}

Kufner M, Gearhart JP, Mathews R

Division of Pediatric Urology, The James Buchanan Brady Urological Institute, The Johns Hopkins School of Medicine, Baltimore, MD, USA

J Pediatr Urol. 2010; 6: 578-81

Objective: Growth of the bladder in children with bladder exstrophy is primarily responsible for later ability to void continently. Improvement in bladder capacity has been noted in some boys following epispadias repair. Does the timing of epispadias repair influence the ability of the bladder to grow?

Methods: Data were collected regarding bladder volume measurements, obtained under anesthesia using a standard technique, during yearly follow-up of boys with classic bladder exstrophy. Volume prior to epispadias repair was compared to the next volume measure following repair. Timing of epispadias repair was compared to changes in bladder capacity in 30 boys. Monthly increases in bladder capacity were calculated in boys repaired at $<12$ (4), 13-24 (12) and 25-48 (14) months.

Results: Patients who had surgery prior to 12 months of age had the highest rate of monthly increase in bladder capacity $(2.40 \mathrm{cc} / \mathrm{month})$. Monthly growth rates were $1.91 \mathrm{cc} /$ month for patients repaired at 13-24 months and $1.18 \mathrm{cc} / \mathrm{month}$ for those repaired at 25-48 months.

Conclusions: Epispadias repair does lead to early increase in bladder capacity in boys with classic bladder exstrophy. The monthly increases in bladder capacity are greater in boys $<12$ months. Improvement in bladder volume is less likely when epispadias is repaired after age 29 months.

\section{Editorial Comment}

This study evaluated the timing of epispadias repair in exstrophy patients with its impact on bladder capacity. The authors retrospectively reviewed all boys undergoing reconstruction, where adequate data were available. The infants underwent routine cystographic evaluation to measure changes in bladder capacity following the initial closure and again 8-16 months following epispadias repair. The authors divided their cohort into three groups: those who underwent epispadias repair prior to 12 months of age; those who were repaired between 13-24 months; and those having reconstruction at 25-48 months of age. They found the greatest increase in capacity over time in those who underwent epispadias repair prior to 12 months of age. Unfortunately, there were only four patients in this cohort. They had larger numbers in the other two groups and both of these showed a trend towards improved bladder capacity with epispadias repair at a younger age.

Although the small number of patients in this study does not lend itself to achieving statistical significance, the data would certainly argue in favor of performing epispadias repair at a younger age. Increasing bladder outlet resistance should improve bladder cycling and allow for improved capacity as the child gets older which will in turn give them the best chance for continence following bladder neck reconstruction. This is the same line of reasoning given by those who favor a complete primary repair at the time of bladder closure.

Dr. M. Chad Wallis

Division of Pediatric Urology University of Utah

Salt Lake City, Utah, USA

E-mail: chad.wallis@hsc.utah.edu 